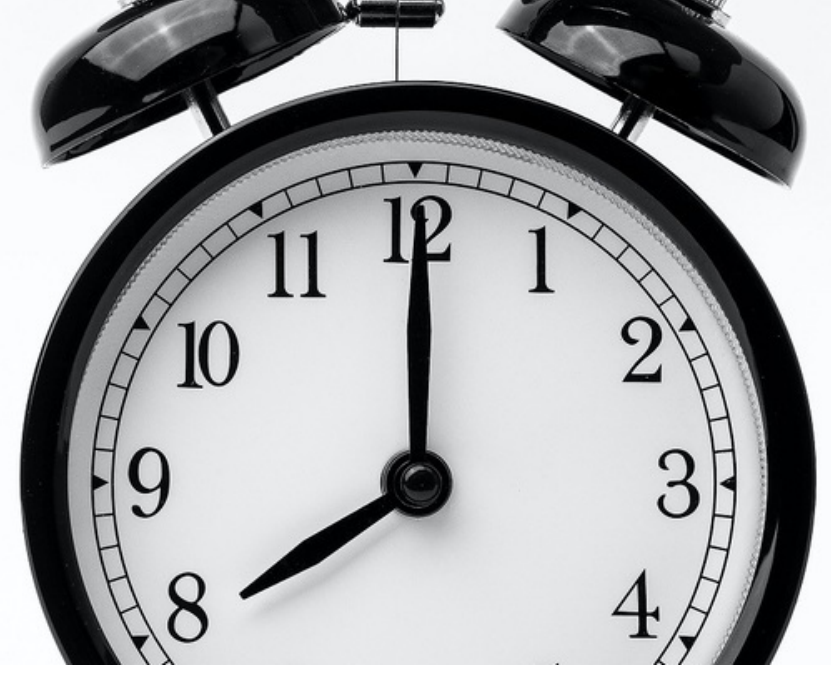

\title{
Abstract:
}

\section{Wie Gefangene im eigenen Zimmer - It's just like being held Hostage in your own Room}

\author{
Authors: Smilla Werle \\ Submitted: $\quad$ 4. May 2021 \\ Published: $\quad$ 17. May 2021 \\ Volume: 8 \\ Issue: $\quad 3$ \\ Affiliation: $\quad$ Walter-Eucken-Gymnasium, Freiburg, Germany \\ Languages: \\ Keywords: \\ Categories: \\ DOI: \\ German \\ Covid, Pandemic, School, Kids, Learning, Homeschooling \\ News and Views \\ 10.17160/josha.8.3.759
}

Since students in Germany have been sent to homeschooling again and again for a year, some problems have developed. Normal lessons have been replaced by a digital device, teachers can only be seen in a small window on the screen and your own room is no longer the place to relax and withdraw. No! The constant lessons in your own room make it difficult to leave the stress of school behind after class and to separate the private from the school. Due to the contact restrictions and the lack of hobbies, students hardly leave the house and spend almost all of their time in their own room. A structured and varied daily routine is not impossible, but it is still superfluous for many students, because every look into the room reminds you of school and this causes stress over and over again.

\section{JOSHA Jounalos semene, Humanities and Arts}




\title{
Wie Gefangene im eigenen Zimmer - It's just like being held hostage in your own room
}

\author{
Smilla Werle
}

\section{Abstract}

Since students in Germany have been sent to homeschooling again and again for a year, some problems have developed. Normal lessons have been replaced by a digital device, teachers can only be seen in a small window on the screen and your own room is no longer the place to relax and withdraw. No! The constant lessons in your own room make it difficult to leave the stress of school behind after class and to separate the private from the school. Due to the contact restrictions and the lack of hobbies, students hardly leave the house and spend almost all of their time in their own room. A structured and varied daily routine is not impossible, but it is still superfluous for many students, because every look into the room reminds you of school and this causes stress over and over again.

Da in Deutschland Schülerinnen und Schüler seit einem Jahr immer wieder ins Homeschooling geschickt wurden haben sich einige Probleme entwickelt. Normaler Unterricht wurde durch ein digitales Endgerät ersetzt, Lehrer sind nur noch in einem kleinen Fenster auf dem Bildschirm zu sehen und das eigene Zimmer ist nicht mehr der Ort, wo man sich entspannen und zurückziehen kann. Nein! Der ständige Unterricht im eigenen Zimmer macht es schwer den Stress der Schule nach Unterrichtsschluss hinter sich zu lassen und Privates von Schulischem zu trennen. Durch die Kontaktbeschränkungen und die fehlenden Hobbies verlassen Schülerinnen und Schüler kaum noch das Haus und halten sich fast ausschließlich im eigenen Zimmer auf. Ein strukturierter und abwechslungsreicher Tagesablauf ist nicht unmöglich aber trotzdem für viele Schülerinnen und Schüler überflüssig, weil jeder Blick ins Zimmer an Schule erinnert und dadurch immer wieder Stress ausgelöst wird. 


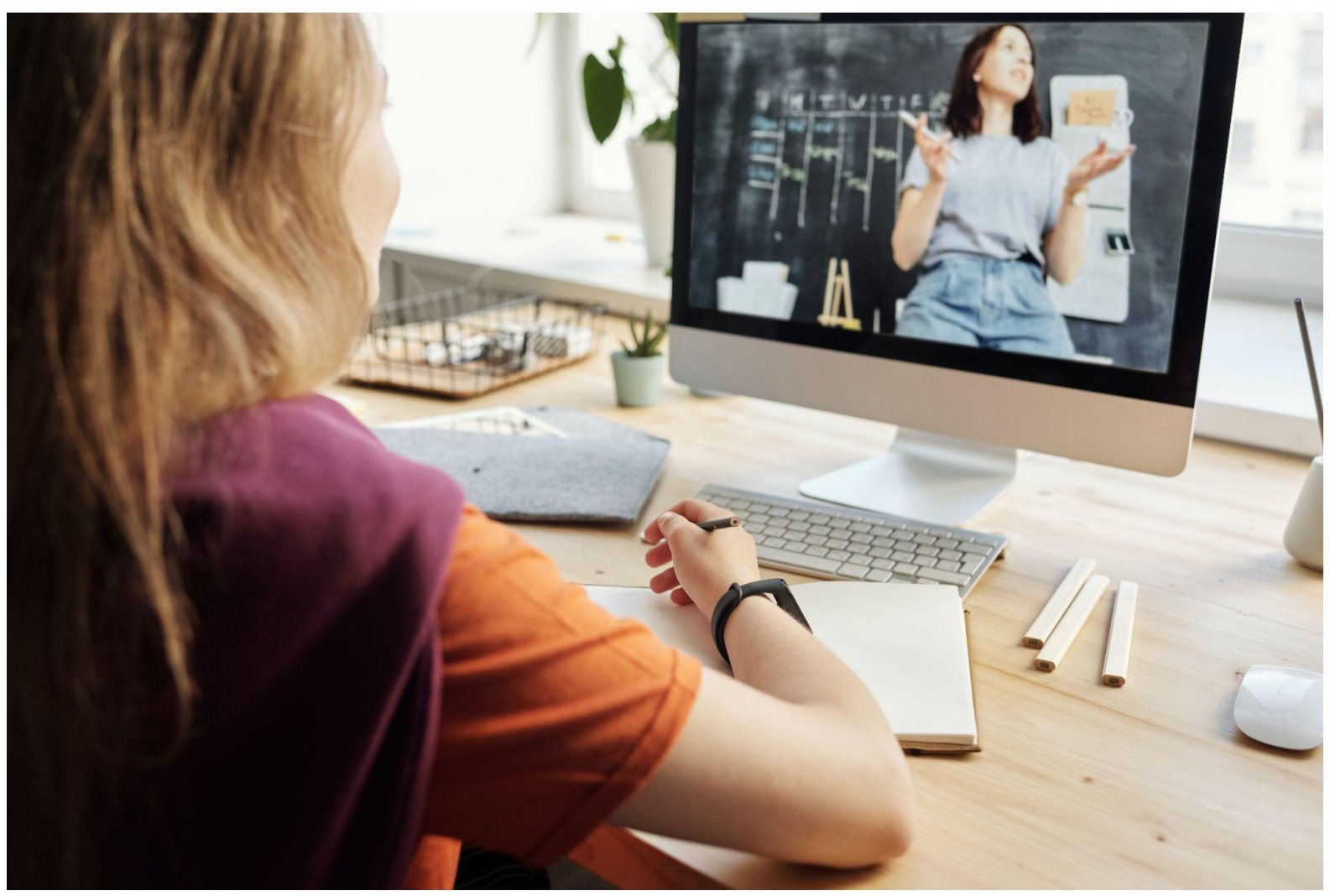

Seit dem 13. März 2020 befinden sich Schülerinnen und Schüler in Deutschland fast ausschließlich im Homeschooling. Seit einem Jahr ist normaler Unterricht nicht mehr vorstellbar. Das, was man früher als Unterricht bezeichnete, wurde durch ein digitales Endgerät in Kinderzimmer, Küche oder Wohnzimmer ausgetauscht. Eltern entwickelten sich zu Lehrern, Spielkameraden und Psychologen gleichzeitig. Ist das der Plan für die nächsten Wochen oder Monate? Wie lange halten die Menschen das durch?

Gemäß einiger Erfahrungsberichte von Schülerinnen und Schüler kann man sich deren Tagesablauf folgendermaßen vorstellen: 7.40 Uhr. Man ist vertieft in seinen Träumen von Freiheit und Weite, Reisen um die Welt und Partys. 7.45 Uhr. Das Erklingen eines schrecklichen Geräusches in den Ohren lässt einen aus dem Schlaf hochschrecken. Durch die fehlende Motivation und dem Schreck des Weckers, der einem tief in den Knochen sitzt, bekommt man als Schüler einen Nervenzusammenbruch, da in einer Viertelstunde der Online-Unterricht beginnt. Völlig übermüdet und demotiviert vom Nichtstun macht man sich auf den Weg zum Schreibtisch, um den Computer hochzufahren. 


\section{Zu viel Bildschirmarbeit und Kommunikationsmangel}

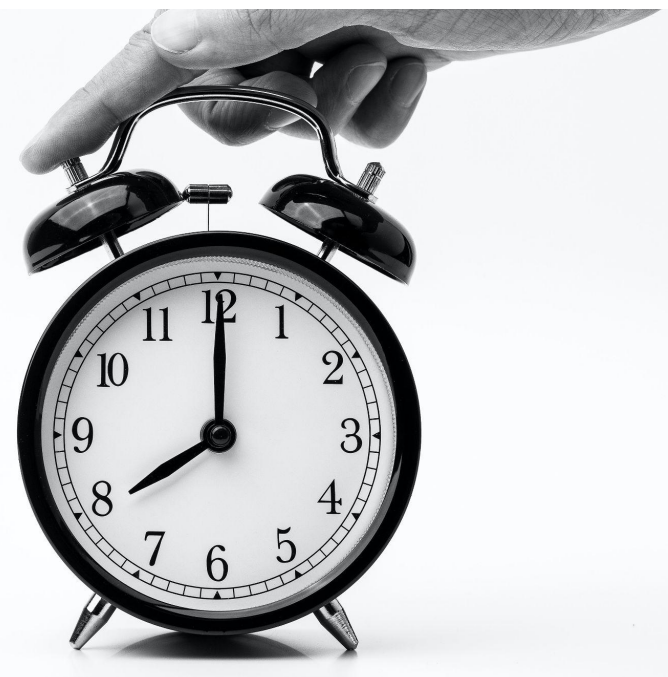

8.00 Uhr. Unterrichtsbeginn. Von nun an sind es ganze lange fünf Stunden bis Unterrichtsschluss, in welchen man sich dem Gerede hilfloser Lehrer aussetzen muss, die gleichermaßen mit den Umständen zu kämpfen haben. Also sitzt man vor seinem Computer, in dem Wissen, dass weitere 30 Menschen dieser endlosen Situation ausgesetzt sind. Um 13.00 Uhr wird gegessen, im Anschluss Aufgaben hochgeladen, so dass man pünktlich um 13.45 Uhr wieder vor dem Bildschirm sitzt. Wenn um 15.15 Uhr der Unterricht endgültig zu Ende ist, sind die Schüler müde von den Bildschirmen, dem starren Sitzen und dem Mangel an Kommunikation. Meistens muss noch nachgearbeitet werden, weil Ausdrucke fehlten oder die Online-Verbindung nicht stabil war.

Der Tag neigt sich dem Ende und der Schüler hat sein Zimmer kaum verlassen können. Kleine Gespräche auf dem Weg zur Schule fehlen, Hobbys und Aktivitäten, die sonst Abwechslung und Motivation bieten, sind derzeit völlig ausgeschlossen. Der Höhepunkt der Woche ist das Wochenende, an dem man ausschlafen kann und eventuell das Haus verlässt. Durch das ständige Lernen zuhause hat sich die Raumatmosphäre von einem idyllischen Entspannungsort zu einem Büro entwickelt. Oft kreisen die Gedanken um Schulaufgaben. Da Kontakte zur Außenwelt fehlen, ist man durchgängig mit den Gedanken bei der Schule. Das einstige Kinderzimmer als Spielort, Kommunikationsstätte, Rückzugsort oder Kreativitätswerkstatt hat sich zu einem Raum voller Pflichtgedanken, Noten und 
Stressfaktoren entwickelt. Die Grenze zwischen Schule und Privatem ist verschwommen. Die Möglichkeit zur Abgrenzung und zum Rückzug wurde den Schülerinnen und Schülern genommen. Der Tagesrhythmus ist verzerrt und bietet kaum klare Strukturen und minimiert den Kampfgeist.

Da ist es kaum verwunderlich, dass sich Kinder und Jugendliche im eigenen Zimmer gefangen fühlen. Umso wichtiger wäre es, den Kindern schnellstmöglich wieder persönlichen Austausch zu ermöglichen. Auch in der Schule.

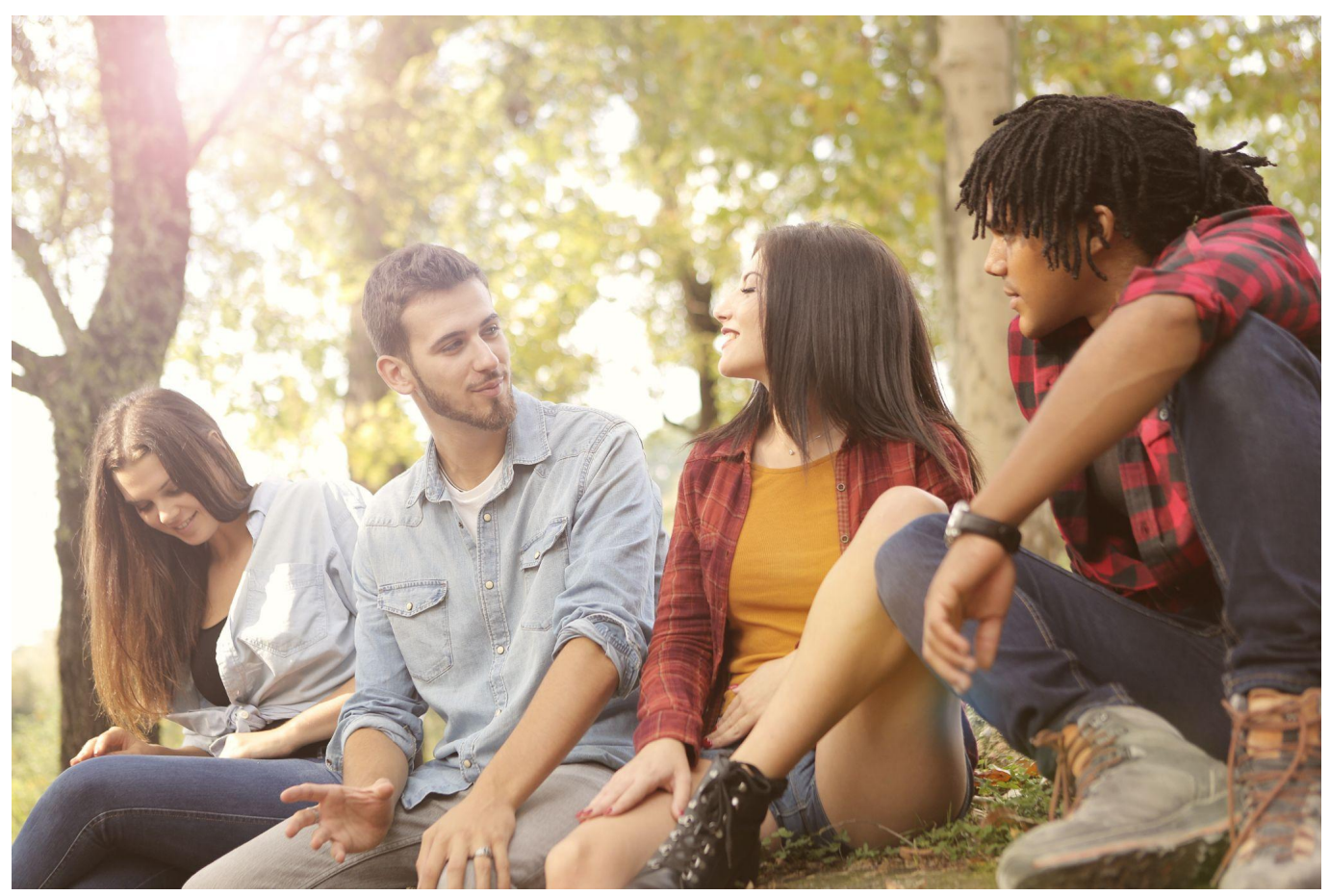




\section{About the Author}

Smilla ist 14 Jahre alt und besucht die neunte Klasse des Walter-Eucken-Gymnasiums in Freiburg.

In ihrer Freizeit zeichnet sie leidenschaftlich gerne und engagiert sich in der Freiwilligen Feuerwehr. 